\title{
EUVAC-Net - the surveillance network for vaccine- preventable diseases is now hosted by ECDC
}

T Derrough (tarik.derrough@ecdc.europa.eu) ${ }^{1}$, A Navarro Torné ${ }^{1}$

1. European Centre for Disease Prevention and Control (ECDC), Stockholm, Sweden

As of 15 September 2011, the European Centre for Disease Prevention and Control (ECDC) will be coordinating the former EUVAC.NET network: a surveillance network for measles, mumps, rubella, congenital rubella, pertussis and varicella in the 27 Member States of the European Union (EU) and in the three countries of the European Economic Area (EEA) [1]. Data will be hosted by the European Surveillance System (TESSy) at ECDC.

EUVAC.NET was established at Statens Serum Institute (SSI, Denmark) in 1999 following an agreement with the European Commission's Directorate General for Health and Consumer Policy (DG SANCO). Since December 2008, EUVAC.NET has been co-funded by SSI and ECDC.

For more than ten years, this well established and recognised surveillance network has provided prominent information on the epidemiology of vaccine-preventable diseases (VPD) in 32 European countries (27 EU Member States together with Croatia, Iceland, Norway, Switzerland and Turkey). It was instrumental in supporting the World Health Organization European Office (WHO-EURO) goal of eliminating measles and rubella in the European Region and will serve as the basis for the continued effort in reaching this target. It has also helped develop the surveillance activities of mumps, pertussis and varicella as well as coordinate efforts for measles, rubella and pertussis laboratory activities. The number of publications and visits to the EUVAC. NET website is a testimony of the success and contribution that this network has had to public health.

ECDC has worked closely with SSI during the transition period to ensure that the high quality and timeliness of data collection are maintained in the new framework of surveillance. ECDC will publish regular comprehensive reports on the epidemiology of the VPD covered by the former EUVAC.NET in order to help guide the prevention and the control in the EU and EEA countries. The widely-used EUVAC.NET website will cease to be active and dedicated webpages will be available on the ECDC website displaying updated surveillance data [1].
A particular feature that was initiated by SSI and will continue is the display of vaccination schedules across the EU. Strengthening and harmonising laboratory activities for pertussis will be part of the new activities ECDC takes on board as well as supporting the development of the WHO-EURO measles and rubella laboratory network.

References

1. European Centre for Disease Prevention and Control (ECDC). EUVAC-Net. European surveillance network for vaccinepreventable diseases. Stockholm: ECDC. [Accessed $14 \mathrm{Sep}$ 2011]. Available from: http://ecdc.europa.eu/EN/ACTIVITIES/ SURVEILLANCE/EUVAC/Pages/index.aspx 